\title{
Characterization of Individual Filaments Extracted From a Bi-2223/Ag Tape
}

\author{
Olaf van der Meer, Xueyu Cai, Jianyi Jiang, Ron D. Parella, Yibing B. Huang, David C. Larbalestier, \\ Bennie ten Haken, and Herman H. J. ten Kate
}

\begin{abstract}
Previous studies suggest that there is a significant variation in both shape and physical properties from filament to filament in a multifilament superconducting Bi-2223/Ag tape. We are investigating this variation for a tape with a high critical current density $\left(J_{c}\right)$.

For this study, we extracted all the filaments from a specially prepared high-quality tape having $J_{c}(0 \mathrm{~T}, 77 \mathrm{~K})=45 \mathrm{kA} / \mathrm{cm}^{2}$ with 19 disconnected filaments. By attaching current leads and voltage taps to the filaments, we are able to measure electrical properties on a scale of 200-300 $\mu \mathrm{m}$.

The experiments show a large variation in filament properties. The conductivity of a filament at room temperature is proportional to its critical current density $J_{c}$ at $77 \mathrm{~K}$ (self-field) and filaments with a higher transition temperature $\boldsymbol{T}_{c}$ do also show a higher $J_{c}$. There is also a variation in the magnetic field behavior of the filaments.

SEM pictures of the filaments also show large variations. The filaments from the center of the tape, which are thinner and have a larger aspect ratio (and thus a larger silver-filament interface), show better grain alignment and fewer impurities than filaments from the edges.
\end{abstract}

Index Terms-Bi-2223/Ag tapes, characterization of filaments, distribution in tape properties, SEM analysis.

\section{INTRODUCTION}

$\mathbf{I}$ N APPLICATIONS like power cables, transformers, motors and generators, superconducting tapes can be used. These tapes, produced by the powder-in-tube method, consist of several $\mathrm{Bi}_{2} \mathrm{Sr}_{2} \mathrm{Ca}_{2} \mathrm{Cu}_{3} \mathrm{O}_{x}$ filaments, embedded in a silver matrix.

During the production process of such a tape, it is exposed to several mechanical deformation steps. The originally round wire is transformed into a tape, by several drawing and rolling steps. Due to these mechanical deformations, the properties of the filaments are not uniform. In the central part of the tape (this is the most deformed part), the filaments are usually wider and thinner than at the edges.

Manuscript received August 5, 2002. This work was supported by the US Department of Energy and by the Dutch Foundation for Research on Matter (FOM).

O. van der Meer is with the Low Temperature Division, Department of Physics, University of Twente, 7500AE Enschede, The Netherlands, and also with the Applied Superconductivity Center, University of Wisconsin Madison, Madison, WI 53706 USA (e-mail: o.vandermeer@tn.utwente.nl).

X. Cai, J. Jiang and D. C. Larbalestier are with the Applied Superconductivity Center, University of Wisconsin - Madison, Madison, WI 53706 USA.

R. D. Parella and Y. B. Huang are with American Superconductor Corporation, Westborough, MA, USA.

B. ten Haken and H. H. J. ten Kate are with the Low Temperature Division, Department of Physics, University of Twente, 7500AE Enschede, The Netherlands.

Digital Object Identifier 10.1109/TASC.2003.812053
Previous studies suggest that there is a significant variation from filament to filament in a multifilament superconducting Bi-2223/Ag tape. In [1] all the filaments from a 7-filament tape were investigated. Among other things, phase purity and texture were studied by x-ray diffraction analysis, while the transition temperature $\left(T_{c}\right)$ and critical current density $\left(J_{c}\right)$ were measured and calculated from magnetization measurements. Cai et al. performed electromagnetical measurements on filaments by attaching current and voltage leads [2]. In this experiment not the complete cross section of the filaments was investigated, because approximately half the filament was cut away, in order to prevent burn-up.

In this contribution, the filaments of a high-quality 19-filament tape are studied. This tape was produced using a standard process. Detailed SEM analysis on several filaments was done, in order to investigate grain structure, grain alignment and the amount of secondary phases/holes. SEM analysis has been performed on both the top view of the filaments and their transverse cross section.

Also the electromagnetical properties of the filaments were studied. This was done as a function of the position of the filament in the tape. The resistivity of the filaments has been measured from room temperature down to the transition temperature. The voltage-current characteristics have been measured in perpendicular magnetic field from 0 to $200 \mathrm{mT}$.

The relation between the resistivity of a filament at room temperature and the critical current density (self-field, $77 \mathrm{~K}$ ) has been analyzed. In [3] several monofilamentary tapes were studied and it was found that if these two quantities are multiplied, a constant value is found. In our experiments, qualitatively the same results have been found, although the constant indifferent.

\section{EXPERIMENTAL DetaILS}

\section{A. Tape Properties}

A specially prepared 19 -filament $\mathrm{Bi}-2223 / \mathrm{Ag}$ tape with low filling factor of approximately $11 \%$ was used in the experiments. Due to this low filling the filaments in the tape are unbridged, which makes it possible to extract all the filaments from the tape. Fig. 1 shows the transverse cross section of the tape. The tape is $2.2 \mathrm{~mm}$ wide and the thickness is $0.15 \mathrm{~mm}$. The critical current density of the tape, measured at $77 \mathrm{~K}$ in self-field, is $45 \mathrm{kA} / \mathrm{cm}^{2}$.

The width of the individual filaments varies between 200 and $400 \mu \mathrm{m}$ and the thickness varies between 10 (for the widest filaments) and $20 \mu \mathrm{m}$. This means that there is a variation in 


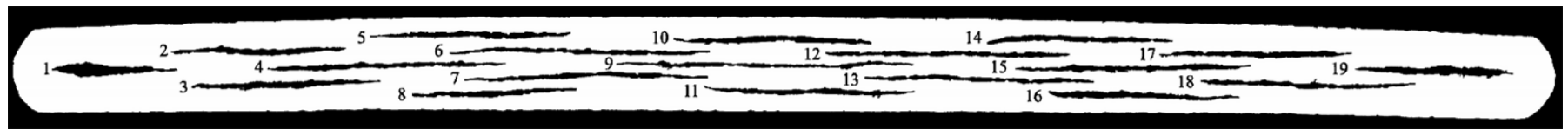

Fig. 1. Cross section of the tape used in the experiments. The silver was etched away and after that the filaments were identified uniquely.

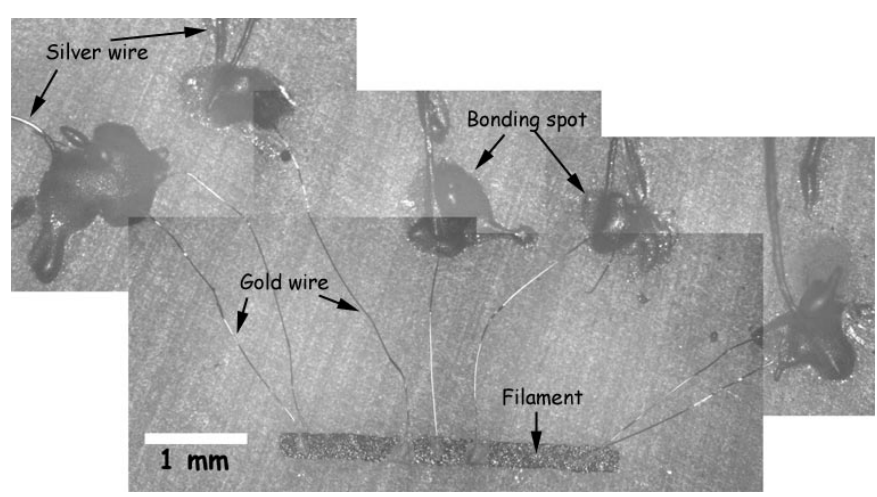

Fig. 2. Optical photograph of a prepared filament. Gold wires connect the filament to a bonding spot (silver epoxy) from which thicker silver wires connect the sample to external circuit.

aspect ratio between approximately 10 and 40. The area of the cross section of the filaments is approximately $1700 \mu \mathrm{m}^{2}$ for all filaments.

\section{B. Preparation of the Samples}

A piece of several $\mathrm{mm}$ long tape was cut under a small angle. Due to this angle the filaments from one side of the tape are longer than the filaments from the other side. All the 19 filaments from this piece were extracted by dissolving the silver sheath in a $\mathrm{NH}_{4} \mathrm{OH} / \mathrm{H}_{2} \mathrm{O}_{2}$ solution. From the length of the extracted filament it is known whether the filament was located in the left, the central or the right part of the tape. By comparing the cross section of an extracted filament, with the cross section of the tape, each filament was identified uniquely.

After the silver is etched away and the filaments are identified, it is possible to prepare the filaments for electrical characterization. The surface of the filament is cleaned by acetic acid, in order to lower the contact resistance between the current leads and the filament.

The filament is placed on a sapphire substrate. On both sides gold current leads $(12.5 \mu \mathrm{m}$ diameter $)$ are attached to the filament using silver epoxy (Fig. 2). These current leads are attached to both the top and bottom of each filament to provide a symmetric current feed into the $a-b$ planes. Between the current leads three voltage leads are attached to the filaments. This enables us to measure two separate sections of approximately 200-300 $\mu \mathrm{m}$.

The gold current and voltage leads are connected, via a bonding spot (silver epoxy) to $50 \mu \mathrm{m}$ silver wires. These silver wires connect the sample to the external circuit for measurements.

\section{Measurement Details}

Resistivity versus temperature measurements of the filaments were performed in vacuum. The resistance was measured using a standard four-point technique with a bias current of $250 \mu \mathrm{A}$.

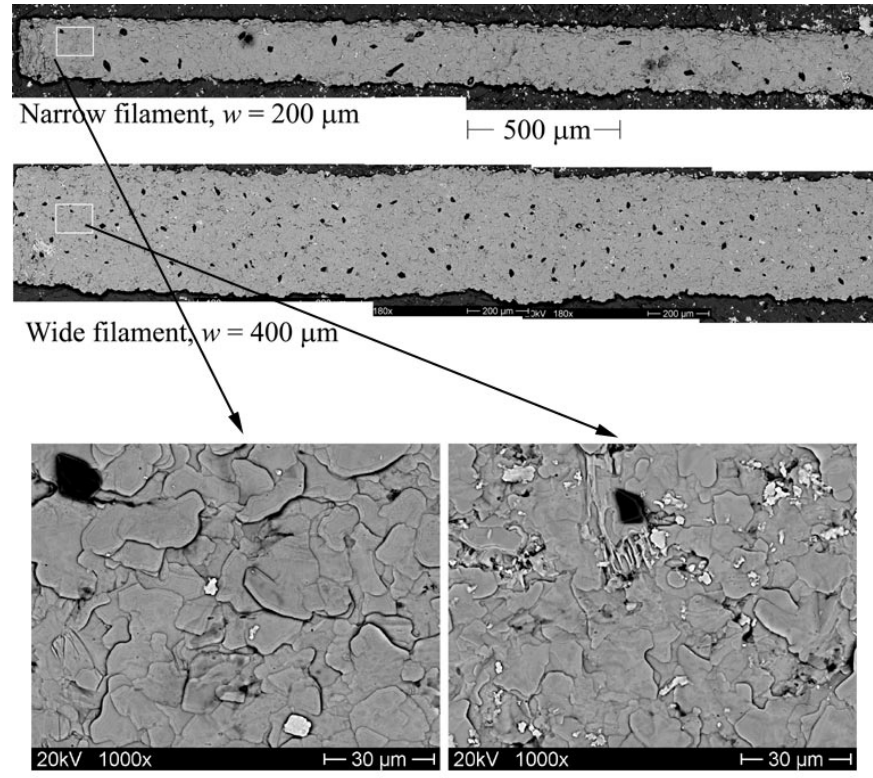

Fig. 3. SEM image of two different filaments. This is a top view of two filaments: a narrow filament from the edge of the tape and a wide filament from the central part. Also a region of each filament is magnified.

The DC voltage-current characteristics of the filaments were measured at liquid nitrogen temperature $(77 \mathrm{~K})$, also using the four-point technique. An electromagnet was used to create magnetic fields up to $200 \mathrm{mT}$. The magnetic field was applied perpendicular to the wide face of the filaments (parallel to the $c$-axis).

The voltage criterion used to determine the critical current density is $1 \mu \mathrm{V} / \mathrm{cm}$. In high magnetic field, the critical current density decreases exponentially [4]. The characteristic field $H_{p}$ is defined by:

$$
J_{c} \propto e^{-H / H_{p}}
$$

For the determination of $H_{p}$, the critical current densities in the region between 70 and $200 \mathrm{mT}$ were used.

\section{SEM STUDY}

Fig. 3 shows a top view of two different filaments. The upper filament is a narrow filament from the edge of the tape and the lower filament is a wide filament from the central part of the tape. The scale for both pictures is the same. In the bottom of the figure, a more detailed part of both pictures is shown. These two pictures have the same magnification.

In the narrow filament fewer black spots are observed than in the wide filament. EDS shows that these black spots are secondary phases $\left[(\mathrm{Ca}, \mathrm{Sr})_{2} \mathrm{CuO}_{3}\right.$ and $\left.\mathrm{CuO}\right]$, so they are not superconducting. However, although the amount of secondary phases is less for the narrow filament, the individual spots are larger. 


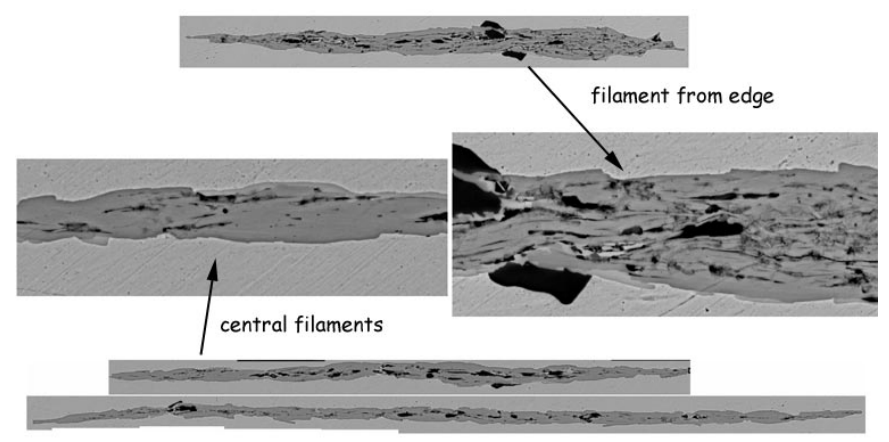

Fig. 4. SEM image of the cross section of the tape. A filament from the edge and two central filaments are shown. Of both filaments a more detailed photograph is shown.

This means that they locally have a larger influence on the total superconducting area and thus on the critical current density.

From the detailed images in the bottom of the pictures it can be concluded that the grain alignment in the wider filament is better than in the narrow filament. In the image of the narrow filament "shadow" lines between grains can be observed more clearly. This means that the angle between the different grains is larger than in the case of the wider filament.

Fig. 4 shows the transversal cross section of different filaments. The upper image shows a narrow filament from the edge of the tape, the lower image shows, on the same scale, two wider filaments from the center. A more detailed part of both images is shown in the middle of the picture.

The detailed images in Fig. 4 show a relatively nice layer of well aligned grains with little secondary phases or holes is seen close to the silver-filament interface. In the central part of the narrow filament a lot of poorly aligned grains can be seen. Also a lot of spots with secondary phases and holes are observed. This is in contrast to the central region of the wider filaments, which has, just like the region close to the interface, relatively well aligned grains. These filaments have a larger silver to filament interface and the distance between the silver interface and the central part of the filament is much smaller.

From the SEM images it can be concluded that the wider and thinner filaments from the central part of the tape are generally better aligned and smaller regions of secondary phases and holes. These filaments have a larger silver to filament interface. Close to such an interface a relatively good aligned region is always observed, also for the narrow and thicker filaments, which have a much less aligned region in the central part.

\section{Electromagnetical MeAsurements}

\section{A. Resistivity Versus Temperature}

In Fig. 5 the resistive transition of several filaments is shown. The filaments have been cooled down in vacuum from room temperature to the transition temperature. The resistance was measured using a current of $250 \mu \mathrm{A}$ through the filament. The transition temperature $T_{c}$ is determined at the point where no measurable voltage was observed. Room temperature resistivities are shown in Table I. In the region between $116 \mathrm{~K}$ and $300 \mathrm{~K}$, the increase in resistivity is almost linear in temperature.

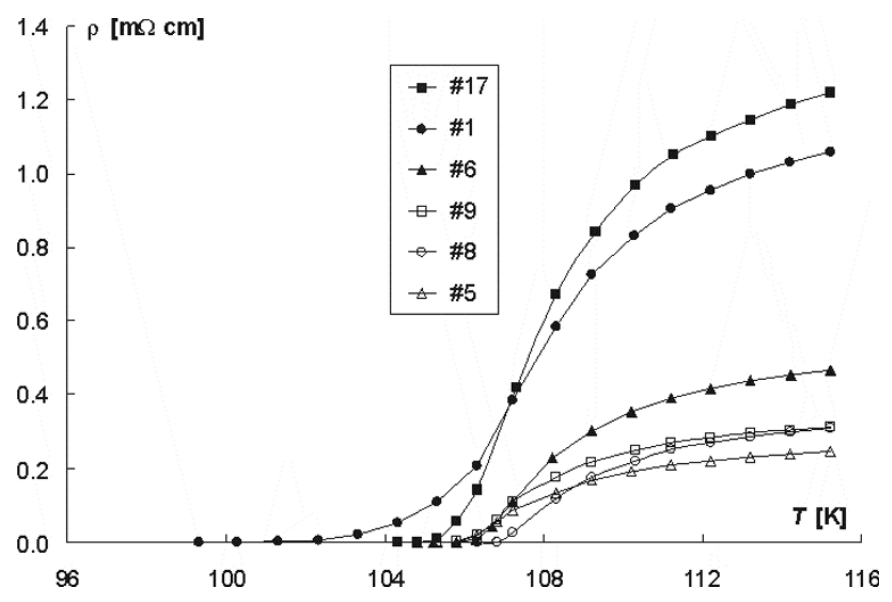

Fig. 5. Resistivity versus temperature measurements on six different filaments. Measurements have been performed from approximately $115 \mathrm{~K}$ down to the transition temperature.

TABLE I

RESUlTS FROM FILAMENT MEASUREMENTS

\begin{tabular}{ccccc}
\hline \hline Filament & $\begin{array}{c}J_{\mathrm{c}} @ 0 \mathrm{mT} \\
{\left[\mathrm{kA} / \mathrm{cm}^{2}\right]}\end{array}$ & $\begin{array}{c}J_{\mathrm{c}} @ 100 \mathrm{mT} \\
{\left[\mathrm{kA} / \mathrm{cm}^{2}\right]}\end{array}$ & $\begin{array}{c}\rho @ 300 \mathrm{~K} \\
{[\mathrm{~m} \Omega \mathrm{cm}]}\end{array}$ & $\begin{array}{c}\mu_{0} H_{\mathrm{p}} \\
{[\mathrm{mT}]}\end{array}$ \\
\hline 1 & 3.5 & 0.73 & 3.0 & 91 \\
5 & 50 & 7.7 & 0.72 & 95 \\
6 & 18 & 3.7 & 1.5 & 111 \\
8 & 43 & 7.6 & 1.0 & 115 \\
9 & 30 & 4.0 & 0.87 & 88 \\
17 & 6.3 & 0.72 & 3.4 & 86 \\
\hline \hline
\end{tabular}

Filaments 1 and 17, which are located at the edges of the tape (see Fig. 1), have a high resistivity at room temperature. Their $T_{c}$ is low, $101.0 \mathrm{~K}$ and $104.5 \mathrm{~K}$ respectively. The other (more centrally located) filaments have a higher $T_{c}$, of about $105.0-105.5 \mathrm{~K}$. They also have a lower resistivity at room temperature.

\section{B. Critical Current Density Versus Applied Magnetic Field}

The relation between the critical current density of the filaments and the applied magnetic field is shown in Fig. 6. These measurements have been performed in liquid nitrogen in order to supply for sufficient cooling to prevent burn-out. The best filaments (numbers 5 and 8, which are in the central part and close to the surface) have a critical current density that is almost a factor 2 higher than the filaments 6 and 9 (from the interior part). Filament 9 has in the low field regime a higher $J_{c}$ than filament 6 , but due to a lower $H_{p}$, the two filaments have a comparable $J_{c}$ at higher magnetic field.

Filaments from the edges (with the lowest critical temperature) also show the lowest critical current densities. This critical current density is a factor 8-10 lower than for the best filaments.

The behavior of all the filaments in the field region between 100 and $200 \mathrm{mT}$ is very similar. There is a spread in $H_{p}$ values from approximately $\mu_{0} H_{p}=85$ to $115 \mathrm{mT}$. As already pointed 


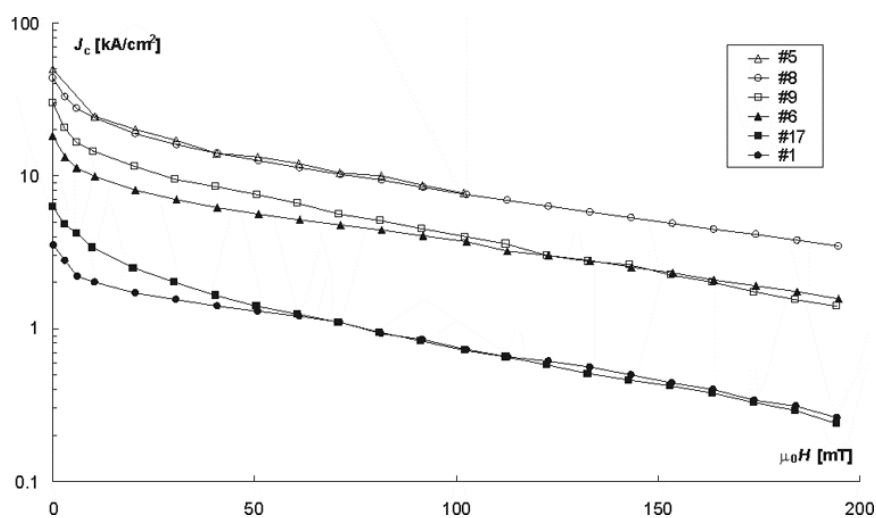

Fig. 6. The critical current density as a function of the applied magnetic field for several filaments. The magnetic field is applied perpendicular to the filament surface.

out, this is best seen in Fig. 6 for the filaments 6 and 9, where the curves cross each other due to different $H_{p}$ values.

\section{Relation Between Resistivity and Current Density}

In Fig. 7 the resistivity of the filaments at room temperature is plotted as a function of the inverse value of the critical current density at liquid nitrogen temperature. Grasso et al. found a linear relation between these two quantities. In that experiment several monofilamentary tapes from the same initial compound, but with different heat treatments/rolling steps were used [3]. These results are also shown in Fig. 7.

In our measurements a linear relation is also observed, although our line does not fit on the Grasso line. This means that there is no "universal" line on which any filament fits. Filaments from our experiment with a certain resistivity have a higher $J_{c}$ than those of the Grasso study with the same resistivity.

The relation between the resistivity at room temperature and the critical current density can be understood as follows: a filament with low resistivity at room temperature has, by definition, good conductivity. This means that the grains are well connected. This good connectivity is also present in the superconducting state. So the $J_{c}$ at $77 \mathrm{~K}$ is also high.

\section{Summary of the Results}

Table I shows a summary of the most important data from the measurements. From Table I it can be concluded that there is a clear correlation between the critical current density and the transition temperature. The best filaments in terms of $J_{c}$ have the highest critical temperatures and vice versa.

There is not always a correlation between $J_{c}$ and $H_{p}$. Although the filaments with the lowest $J_{c}$ values have also a low $H_{p}$, there are also filaments that have a relatively high $J_{c}$, but a low value for $H_{p}$ (see for example filament 5).

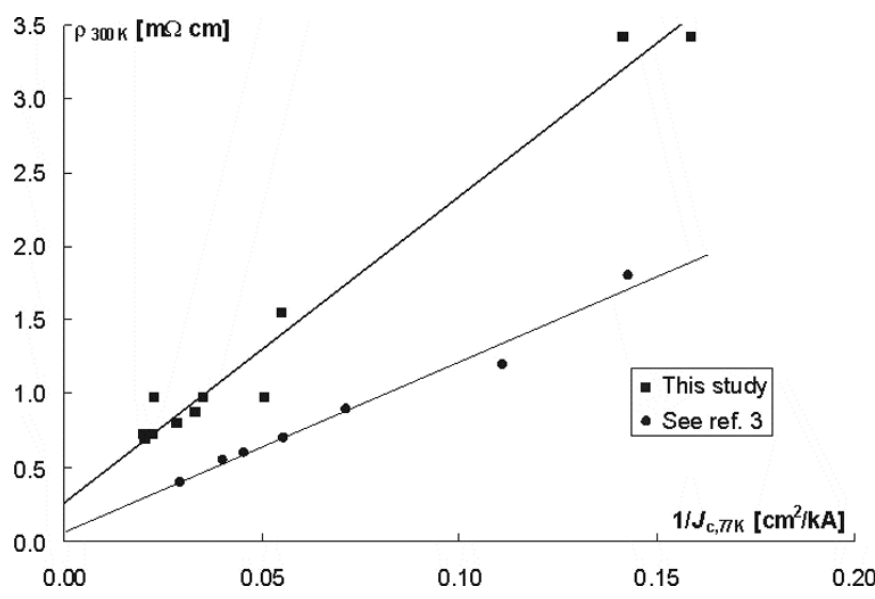

Fig. 7. Relation between the critical current density at $77 \mathrm{~K}$ (in self field) and the resistivity of the filament at room temperature. Also the results from a previous study [3] are included.

\section{CONCLUSION}

There is a large variation in properties of filaments extracted from one single tape. In a tape, there are wider, thinner and narrower, thicker filaments present. From SEM images it can be concluded that there is a well aligned region of grains, close to the silver interface. Images from the top view show that this alignment is better in the wide filaments. Far from the interface there is a much less aligned region, with lots of secondary phases and holes. Because the filaments from the edge of the tape are thicker, there is more material far from the interface. This means that these filaments are the most affected ones.

Electrical measurements show that the filaments from the central part, which have better aligned grains, also have better electromagnetical properties. A clear correlation between transition temperature, critical current density and resistivity is shown. There is no clear correlation between the behavior in higher magnetic fields, characterized by $H_{p}$, and any other parameter.

\section{REFERENCES}

[1] G. Strano, A. S. Siri, and G. Grasso, "Current distribution and microstructure studies of isolated filaments extracted from Ag-sheathed Bi(2223) tapes," Supercond. Sci. Technol., vol. 13, pp. 1470-1475, 2000.

[2] X. Y. Cai, A. Polyanskii, Q. Li, G. N. Riley, Jr., and D. C. Larbalestier, "Current limiting mechanisms in individual filaments extracted from superconducting tapes," Nature, vol. 392, pp. 906-909, 1998.

[3] G. Grasso, F. Marti, Y. Huang, A. Perin, and R. Flükiger, "Correlation between the normal state resistivity and the critical current density of $\mathrm{Ag}$ sheathed Bi(2223) tapes," Physica C, vol. 281, pp. 271-277, 1997.

[4] L. A. Schwartzkopf, J. Jiang, X. Y. Cai, D. Apodaca, and D. C. Larbalestier, "The use of the in-field critical current density, $J_{c}(0.1 \mathrm{~T})$, as a better descriptor of ( $\mathrm{Bi}, \mathrm{Pb})_{2} \mathrm{Sr}_{2} \mathrm{Ca}_{2} \mathrm{Cu}_{3} \mathrm{O}_{x} / \mathrm{Ag}$ tape performance," Appl. Phys. Lett., vol. 75, pp. 3168-3170, 1999. 